\title{
Characteristics of the Intelligent Decision Support System for Precision Medicine (IDSS4PM)
}

\author{
Nasim Sadat Mosavi ${ }^{\text {[0000-0002-6153-2524] }}$ and Manuel Filipe Santos ${ }^{1 \text { [0000-0002-5441-3316] }}$ \\ ${ }^{1}$ Algoritmi Research Centre, University of Minho, Guimaraes, Portugal \\ musavinasimsadategmail.com, mfsedsi.uminho.pt
}

\begin{abstract}
Reducing medical errors, increasing the performance of treatments, cutting the cost of overtreatment, meeting the patient's expectations, and finally save more lives, are some of the major benefits of Intelligent Decision Support System for Precision Medicine(IDSS4PM). This paradigm intends to explore them in the healthcare domain. This study aims to introduce the architecture and remarkable features of such a framework where Simon's model of decisionmaking supports the rationality of the proposed paradigm.
\end{abstract}

Keywords: Intelligent Decision Support System, Medical Decision Making, Precision Medicine, Artificial Intelligence, Analytics, Optimization.

\section{Introduction}

Availability of patient data and promising technologies such as Artificial Intelligence (AI) and analytics have pioneered a new approach in Medical Decision-Making (MDM) [1]. Moreover, increasing the number of chronic diareses and growing the aging population have caused the nations to deal with serious economic pressure. these opportunities and challenges in parallels have motivated the healthcare domain to speed up the transition from a "one-size-fits-all" business model into the "patient-like-me" [6, 31] for tailoring the treatment based on individual patient data.

Whereas, literature has been inspired with so many novel works of applying the latest technologies on healthcare big data [14], Precision Medicine (PM), as a patient-centric approach has been introduced in 2015 to shed light on medical decision-making [11]. Intelligent Decision Support System for Precision Medicine (IDSS4PM) is a framework aiming to support decision making for releasing the right medicine, for the right patient, with the right dose and at the right time. The effective outcome of such practice influences the performance of treatment, and not only results in patient satisfaction but also decreases the costs carries by unsuccessful treatments.

This paradigm is equipped with the particular architecture and features which are discussed in the following sections. First, to present the rationality of this model, Simon's model of decision-making is examined. The concept of Precision Medicine has explained accordingly. After that, the novelty and the major drivers for constructing this paradigm are discussed. Finally, significant features of IDSS4PM are highlighted. 


\section{Simon's Model of Decision-Making}

The "Intelligence-Design-Choice" model, which was identified by Simon, has been applicable as the most complete framework in decision-making. Later he added the "Implementation" phase, which can be explained as a continuous improvement through feedback loops from each phase. As figure 1, demonstrates, "Intelligence" is the first step for scanning the environment to identify problems or opportunities, this phase may include the result of the implementation phase that helps to improve the process of decision-making. "Intelligence" needs attention and attendance; realizing the problem from the symptom of the problem and how should attend the problem needs data collection strategies [28]. Therefore, issues such as availability, quantity, and quality of data and information can challenge this phase [4]. Furthermore, the "Design" phase is about inventing, developing, and analyzing possible courses of actions [17] - In other words, conceptualizing the problem in this phase, leads to modeling where, different scenarios are designed, validate, and tested. This phase needs creativity as well as science to identify the relationship between variables and outlining the hypothesis [4]. Besides, in the third phase, "Choice", there is the matter of evaluating those solutions and choosing among them. But, if the first two jobs have been done well namely, (deciding what to attend to and doing a good job of designing possible courses of action), then in many ways this process of evaluating and choosing would achieve the optimum result [30], In this phase, there is engagement between objectives and model selection. To what extent the particular scenario solves the problem. Moreover, this phase, which is a decision-focused phase, identifies whether the result would be normative or satisfy. In other words, to obtain a normative model, all the possible alternatives, consequences, and value of each, should be considered. This process is called "Optimization"; the highest degree of goal attainment from the available resources. Eventually, the "Implementation" phase is the action step and makes the model work $[4,17]$. These steps are repeated iteratively with many feedback loops until the final choice has been implemented and lessons learned have been identified and communicated [12].

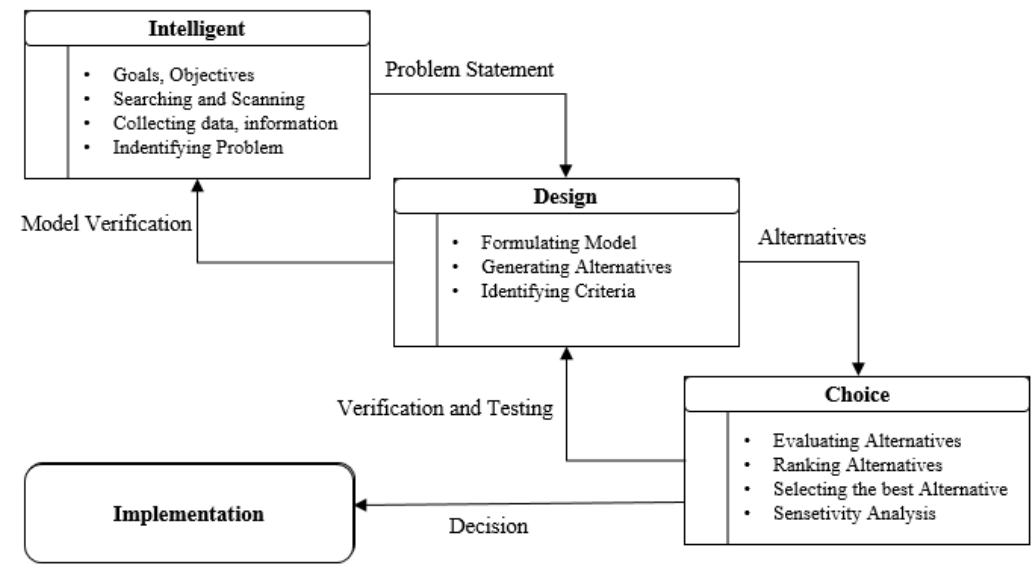

Fig.1. Simon's model of decision-making adapted from [4] 


\section{$3 \quad$ An Emerging Approach in Medical Decision-Making}

\subsection{Precision Medicine}

The traditional practice of MDM, which has been driven by trial and error and physician's judgment, generalizes the solution based on empirical evidence. This approach treats all patients with similar symptoms in the same way [21] and it has caused the patient to become the victim of an ineffective drug or deal with side effects or poor outcomes. Where, answering the questions such as why does a drug work for some patients and be less effective on others? Why does medicine cause side effects on some individuals? needs the medical practice of tailoring each patient as a unique case. On January 20, 2015, the former president Barack Obama launched "Precision Medicine" which has been a remarkable research opportunity in a new area of medical practice [8]. According to the U.S. National Library of Medicine, "Precision Medicine" is an emerging approach that considers individual differences such as genes, environment, and lifestyle for preventing and treating particular diseases [7], [9]. Moreover, the approach of precision medicine is the branch of medical sciences where predicting the possibility of developing a disease, achieving an accurate diagnosis, and optimizing the most effective treatment for a particular patient is the objective [3]. According to figure 2, PM intends to take into account individual patient's variables in terms of genetic, lifestyle, and environmental effects in medical decision-making. Additionally, distinguishing patients from other patients with the same symptoms, improving the performance of treatment, minimizing side effects, and medical [10].

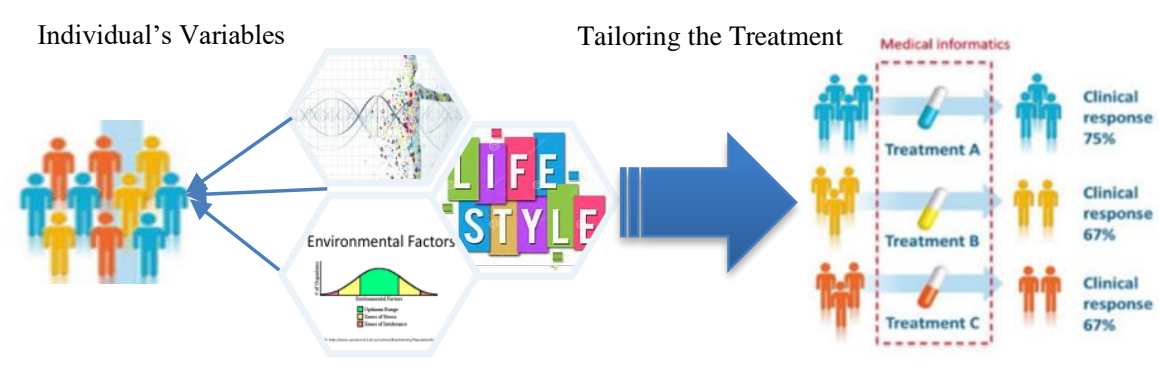

Fig.2. Precision Medicine

\subsection{How Does the Framework Work?}

The availability of patient data which comes from various resources and has been increasingly growing in different formats has challenged the timely decision-making task. Thus limited human cognition is not able to consider all data for releasing the most suitable treatment at the time. Moreover, technological advancement(e.g., analytics, data mining, machine learning algorithms) and powerful methods for characterizing patients (e.g., proteomics, metabolomics, genomics, diverse cellular assays, and even mobile health technology) have inspired automating decision making [7]. 
IDSS4PM, which took advantage of such challenges and opportunities carries the definition of DSS and adopts AI techniques for assisting decision making for releasing precise treatment. Where the new approach in medical decision making is taken into the account. In other words, for deciding about the precise medicine, not only biological factors are important, but also environmental and lifestyle variables are considered.

Because the architecture of this framework is pioneered by the business model of "patient-like-me", so the approach in decision making is patient-centric where the treatment is tailored according to the individual patient variables.

The outcome obtained by IDSS4PM effectively influences the quality of treatment and better performance which results in patient satisfaction and the perceived value of the treatment process. From a macro-economic point of view, this can cut the cost of overtreatment and improve population health [10].

For IDSS4PM to perform effectively, the particular architecture is necessary. According to figure 3, the main drivers of this framework are:

- Individual patient data: which carries the features of healthcare big data (variety, velocity, veracity, value, volume, variability).

- Specific techniques such as clustering for patient segmentation according to the similarity of features.

- $\quad$ Predictive analytics for predicting the features of suitable treatments.

- $\quad$ Prescriptive analytics, which is the most advanced analytics, useful for identifying the best possible arrangement.

- The most suitable technologies to automate decision-making tasks including Artificial Intelligence (AI)/Machine Learning technique, simulation, optimization algorithms, and clustering technique.

Because individual patient data comes from various resources (e.g., business processes, incentive devices, mobility sensors, Internet of Things) and in a different format (e.g., physician notes, nursing notes, surgical notes, radiology notes, pathology reports, admission notes, digitized laboratory slides, radiology videos and images and demographic) [15], therefore patient data is complex, dynamic, semi/unstructured, multi-dimensional and fragmented. Hence, advanced analytics such as predictive and prescriptive analytics are required to perform over the data for providing the best performance of treatment [21].

According to figure3, predictive analytics uses patient past data for predicting the features of the treatment.AI/ Machine Learning techniques are a suitable approach for predicting the most applicable treatments. Furthermore, prescriptive analytics is a decision-focused type of analytics that plays a significant role in determining the result of each treatment path where methods such as simulation and optimization algorithms (e.g., genetic algorithm) are adopted to propose the best possible performance. Moreover, to identify similar patients, techniques such as clustering is necessary to find out and auto-organize similar patients according to the features [4]. 


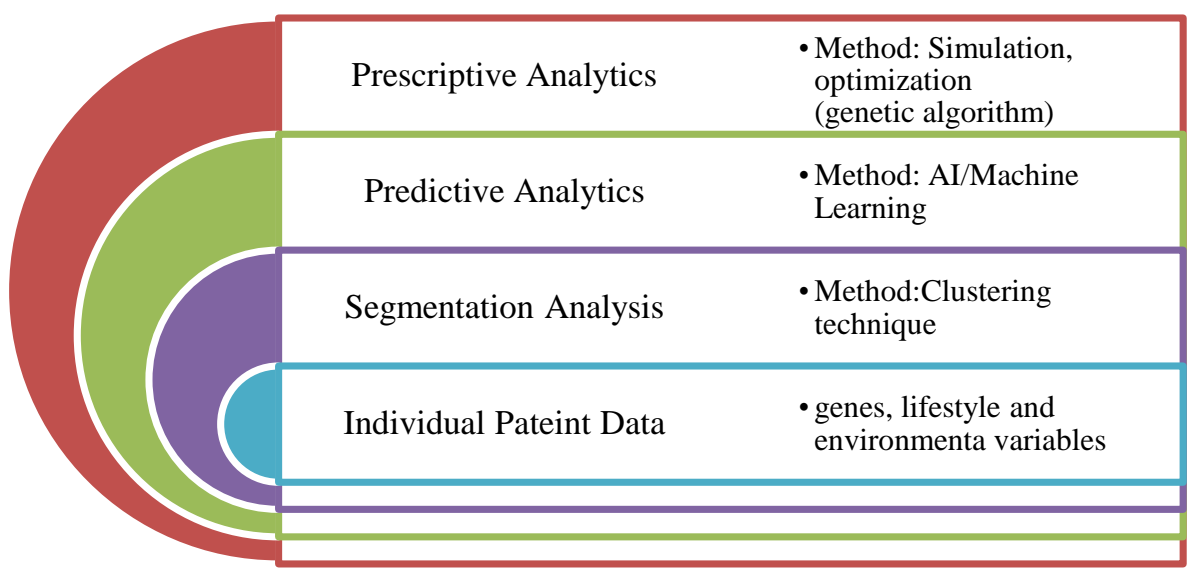

Fig.3. Major drivers for proposing IDSS4PM

\subsection{Features and Architecture}

The IDSS4PM carries major features, which are demonstrated in figure 4.

- Compound DSS architecture

If we consider DSS as a methodology or approach, then IDSS4PM is a computerized application for solving a specific problem in the domain of medicine. Furthermore, because, this framework includes prediction for generating alternatives via AI technology/data mining techniques; it is under the category of knowledge-driven DSS. Knowledge-driven DSSs are useful in the creation of automated decision-making systems. Moreover, because prescriptive analytics and simulation are the main approaches for obtaining the best possible outcome, so the model formulation, model maintenance, model management, and what-if analysis are the foundation of the framework, therefore, it carries the features of a model-driven approach of DSS. Considering this compound architecture (knowledge-driven and model-driven), we can conclude that the hybrid DSS is the most suitable architecture for this framework [22], $[5,23-26]$

- Goal attainment

The solution is goal attainment. In other words, IDSS4PM aims to search, find, and represent the outcome based on goals and the outcome would be the best possible performance to meet the objectives [19, 27].

- Intelligence

The paradigm projects technologies such as artificial neural networks, genetic algorithms, and analytics that carry computational intelligence to construct recommendation algorithms [19].

AI as advanced technology uses the ability of machines to sense, reason, act, and adapt based on learned experience. In other words, AI-based systems work according to the rules and parameters that are fed inside, on the way that machines are capable of making better decisions on the human's behalf [9] Thus, AI is the outcome of computer system based on modeling the human brain to demonstrate intelligent behavior of human brain (e.g., perception, attention, thinking, reasoning) $[2,8,13,16,18,29]$. 
- $\quad$ Adaptive

The framework is dynamic enough to learn from the changes[4]. In other words, the prediction module must be adaptive to make the necessary adjustment and performs according to the continuous improvement [20]

Interactive

Human-computer interaction (HCI) is an important component. Professionals must be able to interact with and define their needs. Moreover, the framework also needs to interact with processors, devices, and cloud platforms [4].

- Contextual

Understanding context is another critical feature. The ability to identify and mining contextual data (e.g., syntax, time, location, requirements, a particular individual's profile) provides flexibility to deal with the integration with multiple resources of data [4].

- Automatic patient segmentation

IDSS4PM uses a clustering technique to group patients based on the variables. This feature selects similar patients according to the major indicators (gene, lifestyle, environmental).

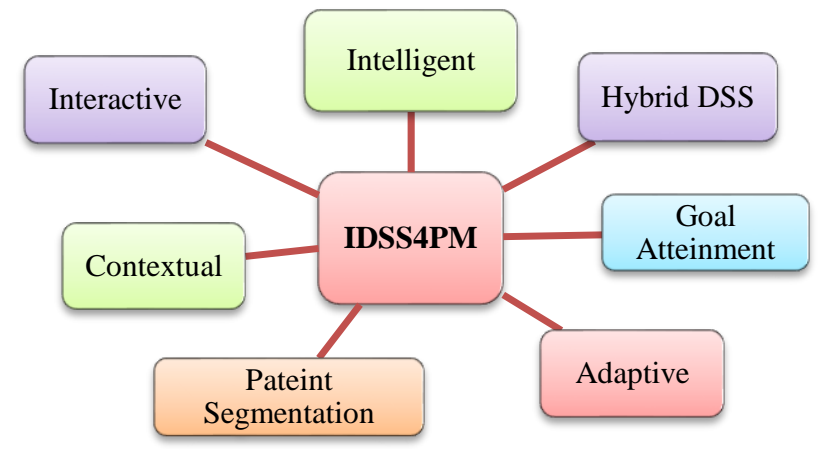

Fig.4. Main features of IDSS4PM

\section{Conclusion}

Failure of the traditional business model: "one-size-fits-all" in addition to the availability of advanced technologies (e.g., AI, machine learning, data mining, analytics) has motivated the healthcare landscape to pioneer a new approach in MDM. This study is inspired by the concept of DSS, which is an umbrella term for providing decisions or recommendations in a problem-solving situation [24].

The IDSS4PM is a new paradigm in Medical Decision Making where Precision Medicine constructs the main objective for obtaining the best possible treatment according to the individual patient data. This paradigm can overcome the challenges of unsuccessful treatment by minimizing medical errors, increasing the quality of treatment, and fulfilling the patient's expectations. Moreover, effective treatment process and saving more lives, strongly result in dropping the costs that are generated as the consequences of the "one-size-fits-all" approach. 
Besides, as it is explained above, according to Simon's model of decision-making, solving a decision-making model in a normative way, includes search approaches (e.g., analytical techniques, algorithms, heuristics, and blind searches), generating alternatives and assessing each alternative and the outcome of each alternative. Furthermore, sensitivity analysis and what-if scenarios are necessary to find the best arrangement of parameters for exploring the changes based on the objectives and goals [24]. Examining this normative approach, the architecture of the IDSS4PM is designed by projecting predictive and prescriptive analytics and using methods such as Machine Learning and optimization algorithms for proposing the most effective and precise treatment for particular patient/s. Hence by applying this theoretical foundation on creating the artifact, it is expected the result is close to an optimal outcome (suboptimal).

\section{Acknowledgments}

The work has been supported by FCT - Fundação para a Ciência e Tecnologia within the Projects Scope: DSAIPA/DS/0084/2018.

\section{References}

[1] Arash Shaban-Nejad, M.M. 2020. Precision Health and Medicine: A Digital Revolution in Healthcare.

[2] Aristodemou, L. and Tietze, F. 2018. The state-of-the-art on Intellectual Property Analytics (IPA): A literature review on artificial intelligence, machine learning and deep learning methods for analysing intellectual property (IP) data. World Patent Information. 55, July (2018), 37-51. DOI:https://doi.org/10.1016/j.wpi.2018.07.002.

[3] Awwalu, J. et al. 2015. Artificial Intelligence in Personalized Medicine Application of AI Algorithms in Solving Personalized Medicine Problems. International Journal of Computer Theory and Engineering. 7, 6 (2015), 439-443. DOI:https://doi.org/10.7763/ijcte.2015.v7.999.

[4] Delen, D. 2020. Prescriptive Analytics The Final Frontier for Evidence-Based Management and Optimal Decision. Pearson Education, Inc.

[5] Felsberger, A. et al. 2017. A review of decision support systems for manufacturing systems. CEUR Workshop Proceedings. 1793, (2017).

[6] Fenning, S.J. et al. 2019. Realistic Medicine: Changing culture and practice in the delivery of health and social care. Patient Education and Counseling. 102, 10 (2019), 1751-1755. DOI:https://doi.org/10.1016/j.pec.2019.06.024.

[7] Francis S. Collins, H.V. 2015. A commentary on "A new initiative on precision medicine." Frontiers in Psychiatry. 6, MAY (2015), 88. DOI:https://doi.org/10.3389/fpsyt.2015.00088.

[8] Gloria Phillips-Wren, N.I. and L.C.J. 2008. Intelligent Decision Making: An AI-Based Approach Studies in Computational Intelligence, Volume 97. Import-01. (2008).

[9] Gupta, S. et al. 2018. Big data with cognitive computing: A review for the future. International Journal of Information Management. 42, April (2018), 78-89. DOI:https://doi.org/10.1016/j.ijinfomgt.2018.06.005.

[10] Hammack, C.M. et al. 2019. Thought Leader Perspectives on Participant Protections in Precision Medicine Research.

[11] Heart, T. et al. 2017. A review of PHR, EMR and EHR integration: A more personalized healthcare and public health policy. Health Policy and Technology. 6, 1 (2017), 20-25. 
DOI:https://doi.org/10.1016/j.hlpt.2016.08.002.

[12] Jatinder N.D. Gupta, G.A.F. et al. 2008. Intelligent Decision-making Support Systems.

[13] Jean-Charles Pomerol 1997. Artificial Intelligence and human decision making. European Journal of Operational Research. (1997). DOI:https://doi.org/10.1145/2987491.2987493.

[14] Jiang, F. et al. 2017. Artificial intelligence in healthcare: Past, present and future. Stroke and Vascular Neurology. 2, 4 (2017), 230-243. DOI:https://doi.org/10.1136/svn-2017000101.

[15] Kaur, J. and Mann, K.S. 2018. AI based healthcare platform for real time, predictive and prescriptive analytics. Communications in Computer and Information Science. 805, (2018), 138-149. DOI:https://doi.org/10.1007/978-981-13-0755-3_11.

[16] Li, J. et al. 2015. Concept learning via granular computing: A cognitive viewpoint. Information Sciences. $\quad$ 298, (2015), 447-467. DOI:https://doi.org/10.1016/j.ins.2014.12.010.

[17] Liu, S. et al. 2010. Integration of decision support systems to improve decision support performance. Knowledge and Information Systems. 22, 3 (2010), 261-286. DOI:https://doi.org/10.1007/s10115-009-0192-4.

[18] Liu, S. et al. 2010. Integration of decision support systems to improve decision support performance. Knowledge and Information Systems. 22, 3 (2010), 261-286. DOI:https://doi.org/10.1007/s10115-009-0192-4.

[19] Lu, J. et al. 2015. Recommender system application developments: A survey. Decision Support Systems. 74, (2015), 12-32. DOI:https://doi.org/10.1016/j.dss.2015.03.008.

[20] Michalewicz, Z. et al. 2006. Adaptive business intelligence.

[21] Palaniappan, S. and Awang, R. 2008. NOTYETUSEDpalaniappan2008. (2008), 108115.

[22] Power, D. 2017. Supporting Decision-Makers: An Expanded Framework. Proceedings of the 2001 InSITE Conference. June (2017). DOI:https://doi.org/10.28945/2384.

[23] Prakash, N. and Sarkar, A. 2015. Development of an intelligent decision support system for an hierarchical business organization. 2015 International Conference and Workshop on Computing and Communication, IEMCON 2015. (2015), 1-8. DOI:https://doi.org/10.1109/IEMCON.2015.7344440.

[24] Ramesh Sharda, Dursun Delen, E.T. 2014. Business intelligence and analytics.

[25] Reza Kheirandish, S.M. 2019. Herbert Simon, innovation, and heuristics. Mind and Society. 17, 1 (2019), 97-109. DOI:https://doi.org/10.1007/s11299-019-00203-6.

[26] Richard, U. and Averweg, F. Decision-making support systems : Theory \& practice.

[27] Schäfer, H. et al. 2017. Towards health (Aware) recommender systems. ACM International Conference Proceeding Series. Part F1286, (2017), 157-161. DOI:https://doi.org/10.1145/3079452.3079499.

[28] Simon, H.A. 1993. Decision Making: Rational , Nonrational, and Irrational. 29, 3 (1993), 392-411.

[29] Skulimowski, A.M.J. 2016. Knowledge, Information and Creativity Support Systems: Recent Trends, Advances and Solutions. 364, February 2016 (2016). DOI:https://doi.org/10.1007/978-3-319-19090-7.

[30] Turpin, S.M. and Marais, M.A. 2004. DeciTurpin, S. M., \& Marais, M. A. (2004). Decision-making: Theory and practice. ORiON, 20(2), 143. https://doi.org/10.5784/202-12sion-making: Theory and practice. ORiON. 20, 2 (2004), 143. DOI:https://doi.org/10.5784/20-2-12.

[31] Williams, A.M. et al. 2018. Artificial intelligence, physiological genomics, and precision medicine. Physiological Genomics. 50, 4 (2018), 237-243. DOI:https://doi.org/10.1152/PHYSIOLGENOMICS.00119.2017. 